\title{
Violência na intimidade: Crenças da comunidade Africana Lusófona residente em Portugal
}

\author{
Mariana Gonçalves ${ }^{1}$, Maria Manuel Silva ${ }^{1}$, \& Marlene Matos $^{1}$ \\ ${ }^{1}$ Centro de Investigação em Psicologia, Escola de Psicologia, Universidade do Minho
}

\begin{abstract}
Resumo: Este estudo teve como objetivo identificar as crenças da comunidade luso-africana, residente em Portugal, sobre a violência na intimidade. Os participantes $(n=70)$ responderam a um questionário online, adaptado da Escala de Crenças sobre a Violência Conjugal. Os resultados apontaram para uma reduzida legitimação da violência, na intimidade, sendo a "pequena" violência a mais legitimada. Os participantes do sexo masculino, sem contacto com a problemática e que avaliaram os episódios como "não graves" apresentaram maior legitimação da violência. Os participantes de zonas urbanas e os residentes há mais tempo em Portugal apresentavam uma menor tolerância à violência. Dos participantes, $17.3 \%$ admitiu ter experienciado violência na intimidade, sendo maioritariamente do sexo feminino. Esta investigação visa contribuir para uma maior consciencialização sobre o tema, para a transformação progressiva dos discursos sobre violência na intimidade e para o desenvolvimento de ações político-legais que visem o combate à elevada ocorrência da violência na intimidade.
\end{abstract}

\section{Keywords: Violência na intimidade; Crenças; Comunidade luso-africana.}

Intimate Partner Violence: Beliefs of the Lusophone African Community Resident in Portugal: This study aims to identify the beliefs of the Lusophone African community, living in Portugal, about intimate partner violence. Participants $(n=70)$ answered to an online survey, adapted from the Scale of Beliefs on Conjugal Violence. The results indicated a reduced legitimation of intimate partner violence, except when it refers to "small" violence. The male participants, those who had no contact with the intimate violence and also those who evaluated the episodes as "not serious" legitimized more intimate violence. Participants that were urban residents and also those who lived longer in Portugal had a lower tolerance to violence. The participants who admitted being victims of intimate partner violence $(17.3 \%)$ were mostly female. This research aims to contribute to a greater awareness about the theme, to the progressive transformation of discourses about violence in intimacy and the development of political-legal actions to combat the high occurrence of violence in intimacy.

\section{Palavras-chave: Intimate partner violence; Beliefs; Lusophone African community.}

A prevalência de violência doméstica ao longo da vida tem sido alvo de vários estudos. Estima-se que $30 \%$ das mulheres em todo o mundo tenham experienciado violência por parceiro íntimo (VPI) durante a vida (Hawcroft et al., 2019). Uma revisão transcultural de aproximadamente 50 trabalhos de investigação realizados em 35 países antes de 1999 revelou que 10\% a 52\% das mulheres relataram ter sofrido abuso físico, abuso sexual ou ambos, perpetrado por um parceiro íntimo durante as suas vidas (Garcia-Moreno et al., 2006). Uma outra revisão sistemática sobre a mesma temática (Alhabib et al., 2010) concluiu que a prevalência da VPI variava de 1,9\% em Washington (EUA) a 70\% em latinas hispânicas no sudeste dos EUA.

A violência doméstica afeta mulheres de todos os grupos étnicos, nacionalidades, sociais e económicos. Em particular, as famílias de imigrantes e refugiados estão em risco de violência doméstica devido à sua história de migração e às diferenças nos valores e normas culturais (Pan et al., 2006). Uma recente revisão sistemática da literatura, que teve como objetivo documentar a prevalência da violência experienciada por mulheres imigrantes no seu país de acolhimento, concluiu que a grande maioria dos estudos concentrou a sua atenção na violência praticada pelo parceiro íntimo, cuja prevalência variava entre $17 \%$ e $70.5 \%$ (Gonçalves \& Matos, 2016). As autoras concluíram que a elevada variabilidade das taxas de prevalência poderá ser explicada não apenas pelas questões metodológicas inerentes aos estudos, mas

\footnotetext{
1 Morada de Correspondência: Mariana Gonçalves, Campus de Gualtar, Rua da Universidade 4710 - 057, Braga, Portugal. E-mail: marianagoncalves@psi.uminho.pt

Este estudo foi realizado em parte no Centro de Investigação em Psicologia (UID/PSI/01662/2013), Universidade do Minho, e foi financeiramente suportado pela Fundação para a Ciência e Tecnologia e pelo Ministérios da Ciência, Tecnologia e Ensino Superior, através de fundos nacionais, e cofinanciado pelo FEDER, através do COMPETE2020, no âmbito do acordo Portugal 2020 (POCI-01-0145-FEDER-007653).
} 
também pelos fatores culturais, uma vez que foram incluídos estudos com amostras heterogéneas, com mulheres oriundas de grupos étnicos diferenciados (e.g., latinas, chinesas, negras).

\section{Crenças sobre a Violência nas Relações de Intimidade}

Alguns fatores socioculturais têm sido identificados como fatores de risco para a VPI, nomeadamente o facto de se pertencer a minorias étnicas, a adesão a normas tradicionais de género e a desigualdade de género, bem como as normas culturais relacionadas com a agressão (WHO, 2014). Por exemplo, uma revisão sistemática da literatura de estudos empíricos sobre os fatores de risco socioculturais para a VPI, em mulheres sul asiáticas imigrantes, concluiu que os principais fatores eram a ausência de apoio social, a reduzida aculturação, a existência de crenças patriarcais, o controlo económico por parte do marido e a presença de atitudes de género tradicionais (Rai \& Choi, 2018).

Sabemos que as normas culturais e sociais assumem um papel importante no comportamento individual, incluindo a violência, podendo por um lado proteger contra a mesma mas, por outro, apoiar e encorajar o seu uso. Por exemplo, a aceitação cultural da violência, seja como um método para a resolução de conflitos ou como uma parte integrante da educação de um filho, é um fator de risco para todos os tipos de violência interpessoal (Krug et al., 2002). Alguns autores defendem que a tolerância social à violência é um comportamento provavelmente aprendido na infância, através o uso de punição corporal (Lansford \& Dodge, 2008) ou do testemunho da violência no seio familiar (Abrahams \& Jewkes, 2005).

0 fenómeno da violência está diretamente associado às representações sociais e o que se considera violento (ou não) tem origem no quadro de referência que cada um possui (Machado, 2010). Mendes e Cláudio (2010, cit. in Ventura et al., 2013) referem que a legitimação da violência é, muitas vezes, consequência de crenças inadequadas que desculpabilizam as condutas abusivas que, sendo interiorizadas desde muito cedo, acabam por influenciar os comportamentos.

Um estudo que analisou a forma como o contexto cultural de 86 mulheres latinas influenciava as suas perceções de violência (Oxtoby, 2012) concluiu que os valores culturais relacionados com os papéis de género estão mais relacionados com as perceções de causalidade da violência, enquanto as atitudes globais sobre a agressão estão mais relacionadas com a aceitação e a gravidade do comportamento violento (Oxtoby, 2012).

Ainda no plano internacional, algumas pesquisas com estudantes e profissionais de saúde têm demonstrado que os participantes tendiam a justificar a violência e acreditavam que as mulheres batidas eram culpadas pelos maus tratos, sendo estas perceções atribuídas à sua ideologia patriarcal e à sua exposição à violência familiar durante a infância e adolescência (Haj-Yahia et al., 2015; Haj-Yahia \& Uysal, 2011). Na área social, Haj-Yahia e Shen (2017) num estudo realizado com 790 estudantes de serviço social de Taiwan verificaram que os estudantes do sexo masculino exibiam uma grande tendência para justificar a violência contra as mulheres e para atribuir a responsabilidade às mulheres vítimas do abuso sofrido. Esta tendência foi também encontrada entre os estudantes que sustentavam atitudes tradicionais em relação às mulheres, que apresentavam expectativas patriarcais sobre o casamento e que tinham testemunhado violência entre os pais na infância.

Já no continente africano, as conclusões vão no sentido de uma maior acentuação das crenças legitimadoras da violência na intimidade. Em Angola, num estudo qualitativo com profissionais de saúde (Nascimento et al., 2014), concluiu-se que as suas perceções eram fortemente marcadas pela construção cultural do papel social da mulher na família, enquanto mãe e esposa, e repletas de preconceitos, fundamentados na pretensa superioridade masculina, atribuindo ao homem uma condição de dominação e à mulher uma condição de ser frágil e desprotegida. Segundo as autoras, as suas visões expressavam as relações hierárquicas de género que geravam a violência, atribuindo à mulher a responsabilidade pelas agressões. Resultados idênticos obteve o estudo qualitativo de Tomás (2016), em Moçambique, nas cidades de Nampula e Maxixe. 0 autor concluiu que a sociedade moçambicana integra o conflito como parte da relação conjugal. Com efeito, as relações conjugais eram relações de poder que traduziam uma dominação e subordinação, envolvendo os conflitos conjugais a imposição da vontade de uns (homem) e a cedência de outros (mulheres). Para as mulheres, o relacionamento conjugal assentava na imposição das vontades dos maridos e na acomodação destas à subserviência. Um estudo mais recente de Neves et al. (2018), com 221 jovens portugueses e 183 jovens cabo-verdianos do ensino secundário, sobre as crenças tradicionais sobre a violência na intimidade e a frequência dos autorrelatos do comportamento violento nas relações de namoro, revelou que os jovens adolescentes cabo-verdianos apresentavam crenças tradicionais mais enraizadas que os portugueses, mas não se verificaram diferenças na prevalência da violência entre os dois grupos. Os autores sugeriram, assim, que as campanhas contra a violência na intimidade não eram tão eficazes em Cabo Verde como pareciam ser em Portugal.

Também em Portugal alguns estudos analisaram as crenças sobre a violência na intimidade. Destacamos o estudo de Machado e colaboradores (2009) sobre as crenças e atitudes dos profissionais de 
saúde, polícias e professores face à violência conjugal. Apesar das conclusões apontarem para uma tendência de discordância moderada face ao conjunto das crenças legitimadoras da violência conjugal, os autores verificaram a existência de crenças desajustadas, tais como "os maus tratos só ocorrem quando há outros problemas dentro da família" (36.4\% de acordo no grupo policial e 9.3\% nos profissionais de saúde) e de que "a causa da violência é o abuso de álcool" (28.3\% no grupo policial e $14.7 \%$ nos profissionais de saúde). Especificamente, entre o grupo policial, os itens que estão ligados à não intervenção externa e ao valor da privacidade familiar reuniram alguma concordância preocupante: "em casos de violência conjugal, a polícia deve apenas tentar acalmar os ânimos e reconciliar o casal" (32.9\%), "a violência conjugal é um assunto privado, deve ser resolvido em casa" (22.4\%, Machado et al., 2009).

Investigações com estudantes (e.g., Caridade, 2011; Machado et al., 2003) concluíram que a generalidade apresentava um nível reduzido de concordância com as crenças legitimadoras da violência, contudo, os estudantes do sexo masculino revelaram um maior grau de tolerância à violência, suportando a crença de que a violência poderá ser justificável face a determinadas condutas da mulher, ou explicada por fatores externos ao agressor (e.g., álcool ou pobreza) e minimizaram mais a "pequena" violência no contexto das relações íntimas.

\section{O Presente Estudo}

Tendo por base a necessidade de investigação empírica sobre este tema, o presente estudo teve como principal objetivo identificar, analisar e discutir as crenças sobre a violência na intimidade sustentadas pela comunidade africana lusófona residente em Portugal. Trata-se de um estudo inovador na medida em que esta problemática, tanto quanto sabemos, não foi ainda alvo de suficiente atenção científica junto desta comunidade, podendo abrir caminho para outros estudos. Para o presente estudo delineou-se como questão de investigação: "Quais são as crenças que a comunidade africana lusófona, residente em Portugal, sustenta sobre a violência nas relações de intimidade?".

\section{MÉTODO}

\section{Participantes}

A amostra era composta por 70 participantes oriundos dos PALOP, sendo a média de idades de 34.72 anos $(D P=12.75)$, variável entre os 18 e os 67 , e o tempo médio de residência em Portugal de 14.46 anos $(D P=$ 13.72), variável entre menos de 1 ano e 43 anos (Tabela 1 ).

\section{Instrumento}

Para este estudo foi revista e adaptada a Escala de Crenças sobre Violência Conjugal (ECVC; Matos et al., 2008), desenvolvida originalmente para avaliar as crenças sobre a violência na intimidade exercida pelos homens contra as mulheres. Essa adaptação consistiu em tornar a escala inclusiva em termos de sexo, ou seja, abordar a temática da violência na intimidade quando esta é exercida pelos homens contra as mulheres e pelas mulheres contra os homens. Por exemplo, refira-se o uso da palavra parceiro(a) em vez de homem ou mulher nas diferentes afirmações. A mudança da terminologia original de "maus tratos" para "violência na intimidade" também foi outra das adaptações efetuadas. A escala dividia-se em 3 partes: parte I - dados sociodemográficos (sexo, idade, nacionalidade (s), país de origem, residência, tempo de permanência em Portugal, estado civil, nível socioeconómico, profissão); parte II - Contacto com a temática; contacto com número de casos de violência na intimidade; formas de conhecimento sobre a temática (ex. formal e/ou informal); e foi vítima ou não de violência na intimidade; parte III - 25 afirmações relativas a crenças sobre a violência na intimidade em que os sujeitos se deveriam posicionar em termos de grau de concordância/discordância. As opções de resposta encontravam-se formuladas numa escala do tipo likert de cinco pontos (1 - discordo totalmente; 2 - discordo; 3 - não concordo nem discordo; 4 - concordo; 5 - concordo totalmente). A escala original revelou uma elevada consistência interna calculada através do coeficiente alpha de Cronbach (.93), sendo que para esta amostra a consistência interna da mesma foi igualmente elevada (alpha de Cronbach $=.91$ ). A análise fatorial demonstrou que esta escala é composta por quatro fatores que explicam $56 \%$ da variância dos resultados: fator 1 - legitimação e banalização da pequena violência, fator 2 - legitimação da violência pela conduta da mulher, fator 3 - legitimação da violência pela sua atribuição a causas externas e fator 4 - legitimação da violência pela preservação da privacidade familiar (Machado et al., 2009). A pontuação total do ECVC pode variar entre 25 e 125, sendo que o fator 1 pode variar de 16 a 80, o fator 2 de 10 a 50, o fator 3 de 8 a 40 e, por último, o fator 4 de 6 a 30. 
Tabela 1. Caracterização sociodemográfica dos participantes $(n=70)$.

\begin{tabular}{llc}
\hline & $\boldsymbol{n}$ & \% \\
\hline Sexo & & 44.3 \\
Masculino & 31 & 55.7 \\
Feminino & 39 & 30 \\
Nacionalidade & 21 & 25.7 \\
$\quad$ Portuguesa & 18 & 21.4 \\
São-Tomense & 15 & 10 \\
Cabo-Verdiana & 7 & 7.1 \\
Moçambicana & 5 & 5.7 \\
Guineense & 4 & 35.7 \\
Angolana & & 28.6 \\
País de origem & 25 & 12.9 \\
São Tomé e Príncipe & 20 & $11-4$ \\
Cabo Verde & 9 & 11.4 \\
Guiné Bissau & 8 & 97.1 \\
Angola & 8 & 2.9 \\
Moçambique & & \\
Residência & 68 & 69.1 \\
Urbana & 2 & 30.9 \\
Rural & & 51.5 \\
Estado civil & 47 & 48.5 \\
Sem relação formal & 21 & 50.7 \\
Com relação formal & & 22.4 \\
Nível socioeconómico & 35 & 26.9 \\
Baixo/Médio Baixo & 33 & \\
Médio/Médio Alto & 34 & \\
Categorias profissionais & 15 & \\
Diferenciada & 18 & \\
Indiferenciada & & \\
Estudantes & & \\
\hline
\end{tabular}

\section{Procedimento}

O questionário foi construído e disseminado online, utilizando o programa Survey Creator, nomeadamente junto de algumas universidades (públicas e privadas), de organizações/associações que trabalham com a comunidade africana residente em Portugal, das redes sociais (exemplo, Facebook) e utilizando a estratégia snowball. Os dados foram recolhidos entre novembro de 2016 e janeiro de 2017.

Os critérios de inclusão eram: ter mais de 18 anos, residir em Portugal e ser originário/a de um país africano lusófono. Todos os participantes foram informados de que a participação era voluntária, tendo liberdade para desistir a qualquer momento da recolha, não envolvendo qualquer tipo de risco ou prejuízo. Foram garantidos os pressupostos éticos e de confidencialidade subjacentes à investigação científica, tendo sido pedida previamente autorização aos autores da ECVC, à editora para que esta pudesse fazer parte do estudo e ser colocada online e, ainda, à Subcomissão de Ética para as Ciências Sociais e Humanas da Universidade do Minho (processo SECSH017/2017).

\section{Estratégia de análise dos dados}

Neste estudo, privilegiou-se o método quantitativo e a análise dos dados foi realizada com recurso ao programa informático Statistical Package for the Social Sciences (SPSS), versão 22.0. Foram utilizadas análises descritivas (frequências e médias) para a descrição sociodemográfica dos participantes e variáveis dependentes. Foram usados testes de diferenças ( $t$ test e ANOVA) para analisar a legitimação da violência em diferentes grupos (e.g., sexo, residência, estado civil, nível socioeconómico, profissão). Correlações de pearson permitiram testar a associação entre a idade e o tempo de residência com a variável dependente ECVC Total. Por fim, para a análise dos subgrupos das vítimas e não vítimas, utilizou-se o teste de associação Qui-Quadrado (Chi-Square Test, $X^{2}$ ), cruzando duas variáveis nominais (vítima ou não vítima e sexo), pretendendo averiguar a existência de uma associação entre elas.

Apesar de terem respondido ao questionário 81 participantes, consideraram-se para a análise 70 participantes, isto é, aqueles que concluíram na íntegra a resposta ao questionário. 


\section{RESULTADOS}

Usando uma análise descritiva para os resultados do ECVC total, verificou-se que a média $(M)$ das respostas foi de $45.06(D P=12.3)$, numa variação de pontuação mínima de 25 e máxima de 74 . Não obstante a tendência de não legitimação da violência a nível dos diferentes fatores, concluiu-se que era sobretudo a "pequena" violência que surgia mais legitimada dentro do contexto das relações íntimas (fator 1), uma vez que foi este fator que apresentou a maior média quando comparadas as médias de todos os outros $(M=$ $27.01, D P=8.0)$. De seguida, surgiu o fator $2(M=18.29, D P=5.44)$, sucedido pelo fator $3(M=15.7, D P=$ 4.92), e, finalmente, o fator $4(M=12.83, D P=4.47)$.

Foram encontradas diferenças significativas entre homens e mulheres ao nível da legitimação da violência, $t(68)=2.64, p=.01$, com os homens a legitimaram mais a violência $(M=49.4 ; D P=13.4)$ do que as mulheres $(M=41.8 ; D P=10.5$; Tabela 2). Foram também encontradas diferenças significativas nos participantes que moravam em zonas urbanas $(M=44.4 ; D P=11.9)$ e rurais $(M=66.0 ; D P=11.3)$, sendo que os residentes em zonas rurais legitimaram mais a violência do que os residentes em zonas urbanas, $t$ $(68)=2.53, p=.014$. Já relativamente ao estado civil, ao nível socioeconómico e à condição de vitimação não se verificaram diferenças significativas.

Tabela 2. Legitimação da violência em função das variáveis sociodemográficas.

\begin{tabular}{|c|c|c|c|}
\hline & $M$ & $D P$ & $t(68)$ \\
\hline Sexo & & & $2.64^{*}$ \\
\hline Homem & 49.4 & 13.4 & \\
\hline Mulher & 41.8 & 10.5 & \\
\hline Residência & & & $2.53^{*}$ \\
\hline Urbana & 44.4 & 11.9 & \\
\hline Rural & 66.0 & 11.3 & \\
\hline Estado Civil & & & -0.24 \\
\hline Com relação formal & 44.5 & 14.3 & \\
\hline Sem relação formal & 45.3 & 11.6 & \\
\hline NSE & & & 0.57 \\
\hline Baixo/Médio Baixo & 46.1 & 10.4 & \\
\hline Médio/Médio Alto & 44.3 & 13.6 & \\
\hline Vítima & & & 1.05 \\
\hline Sim & 41.7 & 9.9 & \\
\hline Não & 45.8 & 12.7 & \\
\hline
\end{tabular}

${ }^{*} p<.05$

Usando o coeficiente de Correlação de Pearson $(r)$, verificou-se não existir correlação entre a idade e os valores totais da ECVC, $r=-.07, p=.55$, mas verificou-se existir uma correlação negativa significativa entre o tempo de residência em Portugal e a legitimação da violência, $r=-.38, p=.001$ : participantes residentes há mais tempo em Portugal legitimavam menos a violência na intimidade.

Quando se recorreu a uma análise de variância (ANOVA) unifatorial $(F)$, não se verificaram diferenças significativas entre as categorias profissionais, $F(2,67)=1.76, p=.18$, entre o número de contactos com casos de violência, $F(2,39)=0.59, p=.56$ e entre as formas de conhecimento sobre a temática e a legitimação da violência, $F(2,67)=1.43, p=.25$.

Contrariamente a esses resultados, quando se analisou a legitimação da violência em função de ter tido contacto com a problemática da violência na intimidade, verificou-se a existência de diferenças significativas, $F(2,67)=4.85, p=.01$, as quais se traduziram, através do teste pos-hoc de Bonferroni, na seguinte evidência: os participantes que não tiveram contacto com a problemática $(M=51.6, D P=12.1)$ legitimaram significativamente mais a violência do que os participantes que não se lembravam se tiveram contacto $(M=45.9, D P=10.0)$ e os que tiveram contacto $(M=41.7, D P=11.8)$.

\section{Vitimação e Legitimação da Violência}

Quanto aos participantes que reportaram ter sido vítimas, a nível da duração dos episódios, $F(3,8)=1.23$, $p=.36$, e da frequência, $F(2,9)=0.76, p=0.49$, não se verificaram diferenças significativas entre estas variáveis e a legitimação da violência. Já sobre a gravidade dos episódios, verificaram-se diferenças 
significativas, $F(2,9)=7.07, p=.01$, que se traduziram, através do teste post hoc de Bonferroni, na seguinte evidência: os que avaliaram os episódios de violência como sendo "não graves" $(M=50.00, D P=5.29)$ legitimaram mais a violência do que aqueles que avaliaram a violência como "muito grave" $(M=29.67, D P$ = 4.51), sendo que o grupo que avaliou os episódios apenas como "graves" $(M=43.5, D P=8.04)$ não se diferenciou estatisticamente dos outros dois grupos.

Por fim, criaram-se dois subgrupos: os que se auto-relataram como não vítimas $(n=57 ; 81.4 \%)$ e os que se auto-relataram como vítimas $(n=13 ; 18.6 \%)$. Através do teste de associação Qui-Quadrado, verificaram-se apenas diferenças parcialmente significativas ao nível do sexo, $X^{2}(1)=2.91, p=.08$, ou seja, uma proporção maior de vítimas era do sexo feminino (77\%), sendo que as não vítimas estavam distribuídas de forma equitativa por ambos os sexos ( $51 \%$ mulheres e $49 \%$ homens).

\section{DISCUSSÃO}

O presente estudo teve como objetivo principal analisar as crenças sobre a violência na intimidade numa amostra comunitária Africana residente em Portugal. Quando se analisam os resultados da escala ECVC, em termos totais, estes apontam para uma reduzida legitimação da violência na intimidade por parte desta comunidade. Esta conclusão vai de encontro ao relatório sobre violência baseada no género Gender based Violence (European Commission, 2016), o qual envolveu os 28 países da União Europeia, informando-nos que a maior parte dos participantes (96\%) considerou errada a violência doméstica contra as mulheres. Este resultado é consonante com trabalhos anteriores neste domínio (e.g., Machado et al., 2009; Ventura et al., 2013) uma vez que, em todos eles, e na sua generalidade, os participantes apresentavam um nível reduzido de concordância com as crenças legitimadoras da violência. Esse resultado é encorajador na medida em que nos oferece uma visão otimista e de mudança, levando-nos a acreditar que se está no caminho certo a nível das principais intervenções implementadas na luta contra este enorme flagelo.

Contudo, quando comparamos os nossos resultados com os estudos efetuados em território africano (Nascimento et al., 2014; Tomás, 2016), verificamos uma tendência oposta, na medida em que nesse contexto parece existir uma maior legitimação da violência devido, segundo Taela (2006, cit. in Tomás, 2016), a uma socialização diferenciada com base no sexo, na crença da superioridade masculina, na instituição de normas que concebem a mulher como propriedade do homem e na aceitação da violência como forma de resolução de conflitos. Esta diferença, no que respeita às crenças sobre a violência na intimidade sustentadas neste estudo e nos estudos em território africano, poderá ser explicada por algumas características da atual amostra: pelo tempo médio de permanência em Portugal, por se tratar de uma amostra jovem, por pertencerem um nível socioeconómico médio/alto e a profissões diferenciadas. Estas características poderão ser um indicador de aculturação. Efetivamente, em Portugal tem-se assistido a um investimento cada vez mais elevado ao nível da prevenção da violência e da disseminação de informação sobre a mesma, o que poderá ter resultado numa maior censura social em relação à mesma. Aliás, dando força a este argumento, verificámos que os participantes da Comunidade Africana Lusófona residentes há mais tempo em Portugal apresentavam uma menor legitimação face à violência.

0 processo de assimilação cultural e, por conseguinte, a defesa de crenças defendidas pela sociedade portuguesa parecem já fazer parte da sua realidade vivencial. Poderemos ainda avançar que as campanhas levadas a cabo em Portugal de prevenção e informação/sensibilização surtiram, de alguma forma, efeito, indo de encontro ao estudo de Neves e colaboradores (2018), em que uma das principais conclusões remete para uma maior eficácia em Portugal, em detrimento de Cabo Verde, das campanhas contra a violência desenvolvidas ao longo dos tempos pelos governos e pela sociedade civil.

Relativamente aos fatores específicos de legitimação avaliados, os resultados confirmam a literatura já existente (e.g., Machado et al., 2003, 2009), no que respeita ao tipo de violência mais legitimada no contexto da intimidade: a "pequena violência". Este resultado significa que é importante continuar a trabalhar na prevenção e sensibilização das populações, na medida que se deve também erradicar a aceitação de formas de violência consideradas menores" (e.g., bofetada, insulto). Essa importância relaciona-se com o facto de as formas de agressão mais graves resultarem muitas vezes da escalada de formas "menores" de violência e do facto desse tipo de violência resultar também num impacto significativo na vida das vítimas.

Podemos ainda realçar que os resultados atribuíram um carácter importante ao papel da mulher como culpada e instigadora da violência, na medida que o fator 2 foi o segundo fator mais legitimado. Este dado está de acordo com a literatura, tanto em estudos com a população africana (sociedades patriarcais onde a mulher assume um papel de submissão, sendo culpabilizada pelos comportamentos agressivos do marido), como com a população europeia, sugerindo que a amostra deste estudo poderá ter assimilado influências dessas duas culturas e, consequentemente, essa crença. Também estudos anteriores revelaram que as crenças mais conservadoras estavam relacionadas com a tendência dos homens para culpabilizar as 
mulheres, acreditando que as mesmas beneficiavam e eram responsáveis pelo abuso sofrido (e.g., Haj-Yahia \& Shen, 2017; Haj-Yahia \& Uysal, 2011; Machado et al., 2003). Esses resultados significam que é importante, por um lado, continuar a combater crenças como "as mulheres gostam de sofrer", "são as mulheres que provocam os maridos", atribuindo ao homem um papel ativo nas dinâmicas abusivas, retirando a centralização/foco no comportamento da vítima.

Este estudo suportou a hipótese de que o sexo masculino legitima mais a violência na intimidade que o sexo feminino. Mais uma vez, este resultado está em sintonia com os dados da literatura, em que os participantes do sexo masculino revelaram perceções mais tolerantes e reforçadoras das crenças legitimadoras da violência (e.g., Haj-Yahia \& Shen, 2017; Haj-Yahia \& Uysal, 2011; Machado et al., 2003). Esses resultados significam também que é importante tornar as campanhas de combate à violência mais inclusivas envolvendo tanto homens como mulheres.

Este estudo suporta, ainda, a hipótese de que os participantes residentes em zonas urbanas apresentavam uma menor tolerância à violência. Este dado é corroborado pela literatura através de vários estudos (e.g., Uthman et al., 2009; Waltermaurer et al., 2013; Wang, 2016). Também Tran et al. (2016), num estudo realizado em 39 países, revelaram que a violência contra as mulheres era mais legitimada e justificada socialmente em pessoas residentes em zonas rurais, sugerindo que a informação sobre a violência na intimidade é mais efetiva, tem mais impacto e atinge um maior número de pessoas nas zonas urbanas. Isso significa que a população das zonas rurais, porventura pelo isolamento e menor acesso à informação, deveria beneficiar mais de campanhas locais e de proximidade, informativas e de sensibilização sobre o fenómeno.

0 estudo permitiu-nos concluir que o grupo de participantes que não teve contacto com casos de violência na intimidade legitimou mais a violência. Podemos avançar para uma explicação face a estes resultados, ou seja, aqueles que tiveram contato com casos de violência na intimidade, perceberam, em contexto real, que se trata de uma séria violação dos direitos humanos, de maior empatia com a posição da vítima, para além de assimilarem a gravidade e complexidade do fenómeno. A sua posição assume-se como crítica e não legitimadora dessa conduta. Por outro lado, aqueles que não tiveram contato poderão desconhecer o fenómeno, tendo uma atitude mais legitimadora. Esta explicação não encontra, contudo, suporte nos estudos de Lichter e McCloskey (2004, cit. in Félix, 2012), os quais demonstraram que os jovens em contato com ambientes violentos tendem a legitimar mais a violência, considerando que se pode recorrer à mesma de forma aceitável para resolver os conflitos. Esses resultados significam que é importante continuar a combater o fenómeno, tanto a nível daqueles que estão expostos ao problema, para que reforcem as suas posições de censura, como a nível daqueles que não estão expostos, para que fiquem mais informados, sensibilizados e assumam atitudes e posições censuradoras dessa conduta.

0 estudo revelou que aqueles que avaliaram os episódios como "não graves" legitimaram mais a violência e os que avaliaram a violência como "muito grave" legitimaram menos. Como possível explicação, sugerimos que o fato de legitimarem a violência pode levar a que percecionar o episódio como "menos grave", normalizando-o, minimizando-o e "desculpabilizando-o". 0 fato de não aceitarem e serem menos tolerantes pode levar a que o percecionem como "mais grave", imputando-lhe um carácter mais censurador.

Por fim, no subgrupo das vítimas verificou-se uma maior percentagem de vítimas do sexo feminino. Estes resultados vão de encontro à literatura que assume uma maior percentagem de mulheres como vítimas. Por exemplo, no relatório da administração interna referente a 2019 (MAI, 2020), 76\% de vítimas eram do sexo feminino. Estão também em conformidade com os estudos africanos de Nascimento e colaboradores (2014) e Tomás (2016) em que a vítima é maioritariamente do sexo feminino, legitimando o estatuto social da mulher numa sociedade em que os valores patriarcais e de dominação masculina assumem ainda um estatuto preponderante. Apesar destes resultados e dos estudos de prevalência em África reportarem para uma maior frequência de vitimação nas mulheres, este dado não é exclusivo deste continente. Assim, houve neste estudo uma contínua preocupação em torná-lo inclusivo, procurando sempre dados relativos à vitimação de homens e mulheres. Exemplo desta preocupação foi também a adaptação da escala ECVC de forma a torná-la mais inclusiva.

Como limitações ao estudo podemos referir, para além do número reduzido de participantes e da não generalização destes resultados, a não inclusão da variável escolaridade na parte dos dados sociodemográficos. São vários os estudos que assumem uma correlação entre a escolaridade e o grau de legitimação da violência, ficando, assim, uma variável importante por analisar. Outra das limitações foi o fato de se ter perguntado aos participantes se já foram vítimas, faltando perguntar-lhes se já foram agressores, uma vez que são conhecidas as elevadas taxas de sobreposição desses papéis neste tipo de violência quando se realizam estudos na comunidade (e.g., Machado et al., 2018). Outra limitação foi desconhecermos se a vitimação reportada pelos participantes, quando se assumiam como vítimas, dizia respeito a episódios de violência ocorridos na relação atual ou em anteriores relações. Os participantes que 
se assumiram como vítimas viviam na sua totalidade em zonas urbanas, o que pode enviesar os resultados na medida em que não se obteve o relato de vítimas que viviam em zonas rurais, constituindo, desta forma, mais uma limitação. Por fim, ter sido um estudo realizado online significa só ter envolvido participantes que, por um lado, sabem manusear e lidar com a internet e, por outro, não têm dificuldades em aceder à mesma. São habitualmente pessoas com profissões mais diferenciadas que estão em permanente contato com a internet, conclusão esta corroborada pelos dados sociodemográficos que revelaram que o maior grupo de participantes ocupava profissões diferenciadas.

\section{CONCLUSÃO}

O estudo respondeu à questão de investigação: "Quais são as crenças que a comunidade africana lusófona residente em Portugal sustenta sobre a violência nas relações de intimidade?", identificando e discutindo essas mesmas crenças e contribuindo para um maior conhecimento desta comunidade. Com esta investigação pretendeu-se estabelecer um ponto de partida para estudos com a mesma população-alvo, uma vez que pouca literatura foi encontrada tendo como foco a comunidade africana lusófona residente em Portugal e a encontrada remonta aos anos 90, princípios de 2000. Desde então Portugal assistiu, por um lado, a uma mudança de paradigma (inclusive sobre a natureza do crime) neste domínio da violência na intimidade e, por outro, a dimensão desta comunidade na sociedade portuguesa continuou a ser significativa, fazendo parte de um passado histórico e com um papel ativo e importante na caracterização dessa mesma sociedade. Assim, o aspeto inovador desta investigação esteve no grupo alvo escolhido.

Consideramos ainda que este estudo contribui para uma maior compreensão do fenómeno da violência na intimidade, desta feita junto da comunidade africana lusófona em Portugal, gerando pistas, conhecimentos e informações que possam ajudar a desenvolver e adotar um instrumento de registo no Centro Nacional de Apoio ao Imigrante (CNAI) e Centros Locais de Apoio à Integração de Imigrantes (CLAII) para recolha de informação do número de casos de violência registados nas comunidades imigrantes. Como objetivos últimos deste estudo, pretendeu-se, ainda, contribuir para uma maior consciencialização do tema junto do grupo-alvo, para a alteração progressiva dos valores sociais e mentalidades, contrariando estereótipos existentes que fomentam a violência na intimidade para a mudança dos discursos sobre estas comunidades e, dentro destas comunidades, os discursos sobre a violência na intimidade e, por fim, para o apoio ao desenvolvimento de ações político-legais que visem a combate à elevada prevalência deste problema criminal e de saúde pública.

\section{REFERÊNCIAS}

Abrahams, N., \& Jewkes, R. (2005). Effects of South African men's having witnessed abuse of their mothers during childhood on their levels of violence in adulthood. American journal of public health, 95(10), 1811-1816. https://doi.org/10.2105/AJPH.2003.035006

Alhabib, S., Nur, U., \& Jones, R. (2010). Domestic violence against women: Systematic review of prevalence studies. Journal of Family Violence, 25(4), 369-382. https://doi.org/10.1007/s10896-009-9298- $\underline{4}$

Caridade, S. (2011). Vivencias íntimas violentas: uma abordagem científica. Almedina.

European Commission (2016). Special Eurobarometer 449. Report “Gender-based violence". European Union.

http://ec.europa.eu/commfrontoffice/publicopinion/index.cfm/ResultDoc/download/DocumentK $\mathrm{y} / 75837$

Félix, D. S. (2012). Crenças de legitimação da violência de género e efeitos de campanhas de prevenção: um estudo exploratório [Dissertação de Mestrado, Universidade de Lisboa].

Garcia-Moreno, C., Jansen, H. A. F. M., Ellsberg, M., Heise, L., \& Watts, C. H. (2006). Prevalence of intimate partner violence: findings from the WHO multi-country study on women's health and domestic violence. Lancet, 368(9543), 1260-1269. https://doi.org/ 10.1016/S0140-6736(06)69523-8

Gonçalves, M. \& Matos, M. (2016). Prevalence of violence against immigrant women: A systematic review of literature. Journal of Family Violence, 31(6), 697-719. https://doi.org/10.1007/s10896-0169820-4.

Haj-Yahia, M., \& Shen, A. (2017). Beliefs about wife beating among social work students in Taiwan. International Journal of Offender Therapy and Comparative Criminology, 61(9), 1038-1062. https://doi.org/10.1177/0306624X15621898

Haj-Yahia, M., Sousa, C., Alnabilsy, R., \& Elias, H. (2015). The influence of Palestinian physicians patriarchal ideology and exposure to family violence on their beliefs about wife beating. Journal Family Violence, 30(3), 263-276. https://doi.org/10.1007/s10896-015-9671-4 
Haj-Yahia, M. M., \& Uysal, A. (2011). Toward an integrative theoretical framework for explaining beliefs about wife beating: a study among students of nursing from Turkey. Journal of Interpersonal Violence, 26(7), 1401-1431. https://doi.org/10.1177/0886260510369135

Hawcroft, C., Hughes, R., Shaheen, A., Utsa, J., Elkadi, H., Dalton, T., Ginwalla, K., \& Feder, G. (2019). Prevalence and health outcomes of domestic violence amongst clinical populations in Arab countries: a systematic review and meta-analysis. BMC Public Health, 19, 315. https://doi.org/10.1186/s12889-019-6619-2

Krug, E. G., Dahlberg, L. L., Mercy, J. A., Zwi, A. B., \& Lozano, A. R. (2002). World report on violence and health. World Health Organization.

Lansford, J. E., \& Dodge, K. A. (2008). Cultural norms for adult corporal punishment of children and societal rates of endorsement and use of violence. Parenting: Science and Practice, 8(3), 257-270. https://doi.org/10.1080/15295190802204843

Machado, A., Hines, D., \& Matos, M. (2018). Characteristics of intimate partner violence victimization experienced by a sample of Portuguese men. Violence and Victims, 33(1), 157-175. https://doi.org/10.1891/0886-6708.VV-D-16-00095

Machado, C., Matos, M., \& Moreira, A. I. (2003). Violência nas relações amorosas: Comportamentos e atitudes na população universitária. Psychologica, 33, 69-83.

Machado, C., Matos, M., \& Gonçalves, M. (2008). Manual da Escala de Crenças sobre Violência Conjugal (E.C.V.C.) e Inventário de Violência Conjugal (I.V.C.). Psiquilibrios Edições.

Machado, C., Matos, M., Saavedra, R., Cruz, O., Antunes, C., Pereira, M., Carvalho, C, \& Capitão, L. (2009). Crenças e atitudes dos profissionais face à violência conjugal: estudos com profissionais de saúde, polícias e professores. Ata Médica Portuguesa, 22(6), 735-742. http://dx.doi.org/10.20344/amp.1739

Machado, L. (2010) Crenças e representações sociais dos adolescentes sobre a violência interpessoal [Dissertação de mestrado, Universidade Fernando Pessoa].

Ministério da Administração Interna, Sistema de Segurança Interna. (2020). RASI - Relatório Anual de Segurança Interna 2019. https://www.portugal.gov.pt/downloadficheiros/ficheiro.aspx?v=\%3d\%3dBQAAAB\%2bLCAAAAAAABAAzNDA0sAAAQJ\%2bleAUAAAA\% $3 \mathrm{~d}$

Nascimento, E., Ribeiro, A. P., \& Souza, E. R. (2014). Perceções e práticas de profissionais de saúde de Angola sobre a violência contra a mulher na relação conjugal. Cadernos Saúde Pública, 30(6), 1229-1238. https://doi.org/10.1590/0102-311X00103613

Neves, A. S., Cameira, M., Machado, M., Duarte, V., \& Machado, F. (2018). Beliefs on marital violence and selfreported dating violence: A comparative study of cape verdean and portuguese adolescentes. Journal of Child \& Adolescent Trauma, 11, 197-204. https://doi.org/10.1007/s40653-016-0099-7

Oxtoby, C. (2012). Taking a cultural perspective on intimate partner violence. [Unpublished Doctoral Thesis. Marquette University].

Rai, A., \& Choi, Y. J. (2018). Socio-cultural risk factors impacting domestic violence among South Asian immigrant women: A scoping review. Aggression and Violent Behavior, 38, 76-85. https://doi.org/10.1016/j.avb.2017.12.001.

Tomás, A. E. (2016). A violência contra a mulher: Um estudo de caso nas cidades de Maxixe e de Nampula [Tese de Doutoramento, Universidade do Porto].

Tran, T., Nguyen, H., Fisher, J., \& Mortimer, K. (2016). Attitudes towards intimate partner violence against women among women and men in 39 low- and middle-income countries. PLoS ONE 11(11): e0167438. https://doi.org/10.1371/journal.pone.0167438

Uthman, O.A., Lawoko, S., \& Moradi, T. (2009). Factors associated with attitudes towards intimate partner violence against women: a comparative analysis of 17 sub-Saharan countries. BMC International Health and Human Rights, 9, 14. https://doi.org/10.1186/1472-698X-9-14

Ventura, C. A., Frederico-Ferreira, M. M., \& Magalhães, M. J. (2013). Violência nas relações de intimidade: renças e atitudes de estudantes do ensino secundário. Revista de Enfermagem Referência, 11, 95-103. https://doi.org/10.12707/RIII12120

Waltermaurer, E., Butsashvili, M., Avaliani, N., Samuels, S., \& McNutt, L.A. (2013). An examination of domestic partner violence and its justification in the Republic of Georgia. BMC Women's Health, 13, 44. https://doi.org/10.1186/1472-6874-13-44

Wang, L. (2016). Factors influencing attitude toward intimate partner violence. Aggression and Violent Behavior, 29, 72-78. https://doi.org/10.1016/j.avb.2016.06.0

World Health Organization. (2014). Global status report on violence prevention 2014. World Health Organization. 
Historial do artigo Recebido

Aceite

Publicado online

Publicado
$01 / 2019$

$03 / 2021$

$04 / 2021$

$06 / 2021$ 